\title{
Alergia e intolerancia a antiinflamatorios no esteroides: desensibilización exitosa en tres casos y revisión de la literatura
}

\author{
Ricardo Cardona, Ruth Helena Ramírez, Zulma Reina, Mauricio Fernando Escobar,
} Edison Morales

\begin{abstract}
Facultad de Medicina, Universidad de Antioquia, Medellín, Colombia. Institución donde se llevó a cabo el trabajo: Clínica León XIII, IPS Universitaria, Universidad de Antioquia, Medellín, Colombia; Clínica Las Vegas, Medellín, Colombia.

Los antiinflamatorios no esteroides son la segunda causa de reacciones a medicamentos después de los beta-lactámicos. La prevalencia de las reacciones a antiinflamatorios no esteroideos en la población general es de $0,1 \%$ a $0,3 \%$ y la prevalencia de anafilaxia inducida por ellos es de $0,01 \%$.

La intolerancia al ácido acetilsalićlico o a los antiinflamatorios no esteroides se presenta con síntomas como urticaria aguda, angioedema, crisis asmática, enfermedad respiratoria exacerbada por ácido acetilsalicílico y anafilaxia.

Presentamos una revisión actualizada basada en tres casos de pacientes con intolerancia al ácido acetilsalicílico o a los antiinflamatorios no esteroideos que, por sus enfermedades concomitantes, requerían tratamiento permanente conácido acetilsalicílico. Después de someterlos a un protocolo de desensibilización rápida ácido acetilsalicílico en una unidad de cuidados intensivos, estos pacientes pudieron continuar recibiendo ácido acetilsalicílico diariamente sin efectos adversos.
\end{abstract}

Palabras clave: hipersensibilidad a los medicamentos, aspirina, agentes antiinflamatorios no esteroides, desensibilización inmunológica, asma, anafilaxis, pólipos nasales, sinusitis.

Allergy and intolerance to nonsteroidal antinflammatory drugs: successful desensitization in three cases

Adverse reactions to nonsteroidal antinflammatory drugs are the second cause of reactions to drugs after beta-lactams. Prevalence of these reactions among general population is between $0.1-0.3 \%$, and prevalence of nonsteroidal antinflammatory drug-induced anaphylaxis is $0.01 \%$.

Acetylsalicylic acid or nonsteroidal antinflammatory drug sensitivity can be manifested as acute urticaria, angioedema, acute asthma, acetylsalicylic acid exacerbated respiratory disease and anaphylaxis.

An updated review based on three cases of patientes with acetylsalicylic acid or nonsteroidal antinflammatory drug intolerance is presented in which, because of their concomitant diseases, permanent treatment with acetylsalicylic acid was required. After undergoing a fast acetylsalicylic acid desentization protocol in an intensive care unit, these patients were able to receive daily acetylsalicylic acid doses without any adverse effects.

Key words: Drug hypersensitivity; aspirin; anti-inflammatory agents, non-steroidal; desensitization, immunologic; asthma, anaphylaxis, nasal polyps, sinusitis.

\section{Correspondencia:}

Ricardo Cardona, Servicio de Alergología, Clínica León XIII, IPS Universitaria, carrera 51B № 69-13, Bloque I, Consultorio 113, Medellín, Colombia.

Teléfono: (574) 516 7300, extensión 3546; fax: (574) 3525288

ricadona@medicina.udea.edu.co, ricardona@une.net.co

Recibido: 21/08/08; aceptado:11/12/08
Los antiinflamatorios no esteroides (AINE) son reconocidos entre los agentes terapéuticos más frecuentemente usados en todo el mundo, con un consumo estimado de 80 tabletas por persona al año. Debido a esta magnitud de exposición, no es sorprendente que los AINE sean una de 
las principales causas de reacciones adversas a productos farmacéuticos en la población (1).

Los AINE son la segunda causa de reacciones adversas a medicamentos después de los antibióticos beta-lactámicos (2). La prevalencia de las reacciones adversas a AINE en la población general varía de $0,1 \%$ a $0,3 \%$ (2) y hasta $0,9 \%$ en otras series (3). En un estudio retrospectivo realizado entre 1985 y 1996, en un hospital universitario de Florencia (Italia), se recolectó la información de los pacientes que ingresaron al servicio de urgencias, con un total de 153.086 admisiones por todas las causas, de las cuales, 113 correspondieron a anafilaxia. Del total de casos de anafilaxia, las causadas por medicamentos fueron las más comunes, con 52 episodios (49\%). De éstos, 25 (48\%) fueron por antibióticos y $18(35 \%)$ por AINE. La prevalencia de anafilaxia inducida por AINE fue de $0,01 \%$ (4).

La intolerancia al ácido acetilsalicílico o los AINE, definida como las reacciones cutáneas (urticaria, angioedema 0 ambas) o respiratorias (rinitis, broncoespasmo o ambas) que se presentan tras la administración de dos o más AINE de grupo químico diferente, ocurre en $2 \%$ a $23 \%$ de los pacientes asmáticos, según sea la población estudiada y el método diagnóstico utilizado (5), en $14 \%$ a $22 \%$ de los casos de poliposis nasal y tan sólo en $0,7 \%$ a $1,4 \%$ de los de rinitis no alérgica (6).

En pacientes con asma grave y poliposis nasal, la incidencia de intolerancia a ASA o AINE puede llegar a $78 \%$ (7), mientras que las urticarias inducidas por AINE se deben a un fenómeno de intolerancia en $75 \%$ de los casos. En estos casos se habla de hipersensibilidad cruzada. Por otro lado, cuando un paciente ha presentado una o más reacciones a un antiinflamatorio no esteroideo AINE en particular o a uno del mismo grupo químico, se habla de hipersensibilidad simple. Estas reacciones se han atribuido a una verdadera reacción alérgica, en la cual la sensibilización inicial es esencial (8).

Los AINE pueden clasificarse de acuerdo con su estructura química o según el grado de selectividad de inhibición de las isoenzimas ciclooxigenasas (COX); esta última clasificación es útil a la hora de definir si el paciente con reacción adversa tras el consumo de AINE, presenta hipersensibilidad simple (alergia) o hipersensibilidad cruzada (intolerancia).

La clasificación según la estructura química considera nueve grupos, así: alkanonas (por ejemplo, nabumetona), ácidos antranílicos o fenamatos (ácido miclofenámico y ácido mefenámico),ácidosarilpropiónicos (fenoprofeno, flurbiprofeno, ibuprofeno, ketoprofeno, naproxeno, oxaprozín), ácidos enólicos (oxicams: piroxicam, tenoxicam; pirazolidinedionas: oxifentatrazona, fenilbutazona), ácidos heteroarilacéticos (diclofenaco, ketorolaco,tolmetín), ácidosindolacéticos (indometacina, etodolaco, sulindac), derivados del para-aminofenol (acetaminofén), pirazolonas (aminopirina , dipirona, antipirina) y salicilatos (ácido acetilsalić́lico, salicilato sódico, salsalato, sulfasalazina, diflunisal) (2).

La clasificación según la selectividad de inhibición de las COX establece cuatro grupos, así: inhibidores débiles de COX (acetaminofén, salsalato, salicilato sódico), inhibidores COX-1/ COX-2 (piroxicam, indometacina, sulindac, tolmetín, ibuprofeno, naproxeno, fenoprofeno, acido mefenámico, meclofenamato, diclofenaco, ketorolaco, etodolaco, nabumetona, flurbiprofeno), inhibidores preferenciales de COX-2 (nimesulide, meloxicam) e inhibidores selectivos de COX-2 (celecoxib, rofecoxib, valdecoxib, parecoxib, etoricoxib, lumiracoxib) (2).

\section{Materiales y métodos}

Los casos que se reportan a continuación se originaron en pacientes de la consulta del Servicio de Alergología de la Clínica de la Clínica León XIII de la IPS Universitaria de la Universidad de Antioquia, en los que se estableció la necesidad de tratamiento con ácido acetilsalicílico para su enfermedad de base, sin otra alternativa terapéutica y que no podían recibir este medicamento por intolerancia, lo que obligaba a realizar un procedimiento de desensibilización.

Cada paciente debía estar estable clínicamente en la semana inmediatamente anterior al procedimiento y usando los medicamentos indicados para el control adecuado de su enfermedad 
de base. La estabilidad clínica pulmonar se establecía mediante espirometría previa en la que se observaba principalmente el volumen espiratorio forzado en el primer segundo $\left(\mathrm{VEF}_{1}\right)$, el que debía estar por encima del $70 \%$ del predicho como condición previa a la realización del procedimiento.

Se estableció como obligatorio para todos los pacientes que el procedimiento de desensibilización se llevara a cabo en la unidad de cuidados intensivos, por la garantía de monitorización y manejo de reacciones sistémicas graves, como podía ser el caso de una reacción anafiláctica.

En todos los casos el protocolo de desensibilización se hizo con un esquema rápido publicado en el 2006 (9), probado como eficaz y seguro en una amplia serie de pacientes. Se usó tanto en el caso que lo requería en el manejo de riesgo en enfermedad cardiovascular, como en los casos de enfermedad respiratoria. El protocolo establece que los pacientes sean monitorizados continuamente en cuanto a sus signos vitales, con control cada 90 minutos de la función pulmonar con espirometría y de la función nasal con medición del pico flujo nasal inspiratorio. Se requería disponibilidad del equipo de reanimación. En todo momento, los pacientes debían tener acompañamiento del equipo de alergología y del personal médico y paramédico de la unidad de cuidados intensivos (10).

Los instrumentos de medición utilizados en todos los procedimientos fueron: para la función pulmonar, un espirómetro marca Vitalograph $\AA$, referencia 2120, debidamente calibrado con jeringa de 3 litros de volumen; para la función nasal, un medidor de pico flujo nasal inspiratorio marca In-Check®, referencia 1902164; para el control de signos vitales en la unidad de cuidados intensivos, se usó el monitor de signos vitales con cardioscopio propio de cada una de las instituciones donde se realizaron los procedimientos.

Se tomó como criterio de desensibilización eficaz, el hecho de tolerar la dosis final del protocolo de 650 mg de ácido acetilsalicílico por vía oral, para los casos de enfermedad respiratoria, y de 100 $\mathrm{mg}$, para el caso de enfermedad cardiovascular. La tolerancia al ácido acetilsalicílico al final del procedimiento se estableció por la ausencia de reacciones evidentes clínicamente, fueran oculares, nasales, pulmonares o cutáneas, además de la ausencia de caída del $V E F$ respecto de la medición basal no mayor de $20 \%$.

La mejoría clínica posterior al procedimiento para las enfermedades que padece cada uno de los pacientes, no es del alcance de este artículo, pero se establecerá a través del tiempo con el control del riesgo de evento mayor cardiovascular, en el caso de la paciente con angina inestable, y el control de la enfermedad rinonasosinusal, para los otros casos presentados.

Caso 1. Se trata de una mujer de 63 años de edad, residente en Medellín, remitida al Servicio de Alergología Clínica por el cardiólogo tratante, porque requería la administración de $100 \mathrm{mg}$ diarios de ácido acetilsalićlico como parte del tratamiento profiláctico para evitar un evento cardiovascular mayor, por padecer angina inestable e hipertensión arterial.

Recibía tratamiento con metoprolol, hidroclorotiazida y lovastatina. Además, tenía el antecedente de reacción adversa al ácido acetilsalicílico, al haber presentado angioedema con su consumo y también historia de urticaria por otros AINE.

Antes del procedimiento de desensibilización al ácido acetilsalicílico, se solicitó nueva valoración por el cardiólogo, quien sugirió el cambio del medicamento antihipertensivo debido a la poca respuesta a la adrenalina en pacientes que reciben beta-bloqueadores y cursan con anafilaxia. También, se le solicitó que considerara la opción de un tratamiento alternativo al ácido acetilsalicílico. El cardiólogo decidió el cambio del antihipertensivo por verapamilo e informó que, en su criterio como especialista, la paciente debía recibir ácido acetilsalicílico, no otro antiagregante plaquetario $u$ otro AINE.

Se llevó a cabo el procedimiento de desensibilización en la unidad de cuidados intensivos, siguiendo un esquema rápido publicado recientemente (9), hasta que toleró una dosis de $100 \mathrm{mg}$ sin presentar reacción. El procedimiento tuvo 
una duración total de 4 horas, no se presentaron reacciones adversas y la paciente fue dada de alta, con control a las 24 horas para evaluar reacciones tardías, las cuales tampoco se presentaron. La paciente continuó tomando 100 mg de ácido acetilsalicílico cada día, con buena tolerancia.

Caso 2. Se trata de una mujer de 51 años de edad con diagnóstico de rinosinusitis crónica, poliposis nasal y sinusal recurrentes, con antecedente de tres polipectomías previas, asma e intolerancia a AINE (urticaria y angioedema con ácido acetilsalicílico, anafilaxia con ibuprofeno y con diclofenaco).

Con el objetivo de ofrecerle una alternativa analgésica y antiinflamatoria, se le realizó previamente una prueba de provocación oral con celecoxib, que fue negativa. Posteriormente, se llevó a cabo una prueba de provocación nasal con ácido acetilsalićlico que no fue concluyente. Se procedió a realizar la desensibilización oral con ácido acetilsalicílico para ofrecerla como tratamiento a largo plazo, con el fin de disminuir la recurrencia de la poliposis y de los episodios de rinosinusitis y lograr un mejor control del asma, como lo han demostrado diferentes estudios considerados en la discusión.

Se estabilizaron los síntomas de asma y rinitis, se vigiló que no tuviera ningún proceso infeccioso activo y se procedió a la desensibilización en la unidad de cuidados intensivos siguiendo un protocolo rápido (9) que establece el suministro por vía oral de dosis crecientes de ácido acetilsalicílico; se inicia con una dosis de 4 $\mathrm{mg}$; a los 90 minutos, se administra una dosis de $40 \mathrm{mg}$ y, a los 90 minutos de esta segunda dosis, una de $81 \mathrm{mg}$; de ahí en adelante, cada 90 minutos se suministra el doble de la dosis anterior, hasta alcanzar una dosis final de 650 $\mathrm{mg}$, que se suministra a las 7 horas y media de haber dado la primera.

En el caso de esta paciente, se administraron dosis progresivas de ácido acetilsalicílico hasta $81 \mathrm{mg}$, cuando presentó un episodio de prurito generalizado, tos y sibilancias sin disminución del volumen espiratorio forzado en el primer segundo $\left(V_{E F}\right)$. Se manejó con un agonista beta-2 inhalado y clemastina. Se reinició el procedimiento 90 minutos después, administrando la dosis inmediatamente anterior, y se continuó hasta que toleró una dosis de 650 mg sin presentar reacción. Se administró una dosis total acumulada de $1.262 \mathrm{mg}$, con buena tolerancia. La paciente fue dada de alta y revisada a las 24 horas para evaluar reacciones tardías, las cuales no se presentaron. Continuó recibiendo una dosis de $650 \mathrm{mg}$ de ácido acetilsalicílico cada 12 horas y la ha tolerado hasta el presente.

Caso 3. Se trata de una mujer de 37 años de edad con diagnóstico de rinosinusitis crónica, asma bronquial de difícil manejo y antecedente de cinco polipectomías nasales e intolerancia a AINE (anafilaxia por ácido acetilsalicílico que requirió ingreso a la unidad de cuidados intensivos). Debido al antecedente de anafilaxia, no se realizaron pruebas de provocación nasal u oral para confirmar el diagnóstico de intolerancia a AINE o enfermedad respiratoria exacerbada por ASA.

Se decidió realizar la desensibilización al ácido acetilsalicílico en la unidad de cuidados intensivos para ofrecerla como tratamiento a largo plazo, con el fin de disminuir la recurrencia de la poliposis y de los episodios de rinosinusitis y lograr un mejor control del asma, como lo han demostrado diferentes estudios considerados en la discusión.

El día del procedimiento, la paciente se encontraba en condiciones clínicas estables, con un $V_{E F}$ semejante al habitual para ella de $71 \%$ del valor predicho. Se administraron dosis progresivas de ácido acetilsalicílico hasta 81 $\mathrm{mg}$, cuando fue necesario suspenderlo por la aparición de prurito en el canal auditivo que posteriormente se generalizó, tos en accesos, descenso de la presión arterial sistólica del 20\% y del $\mathrm{VEF}_{1}$ del $40 \%$ con respecto a los valores basales. Clínicamente presentó lagrimeo, inyección conjuntival, obstrucción nasal y sibilancias, con saturación de oxígeno normal.

Se suspendió el procedimiento y se inició el manejo de la crisis asmática con un esquema de rescate usando salbutamol; se aplicó una dosis 
de clemastina de $2 \mathrm{mg}$ y se vigiló estrechamente durante 90 minutos. Se presentó reversión rápida de los síntomas, pero la recuperación del VEF, fue insuficiente para reiniciar el procedimiento, considerado como parámetro recomendado el tener una pérdida no mayor del $20 \%$ del valor basal.

Se decidió prolongar el esquema de rescate de crisis asmática usando salbutamol por 24 horas, y retomar el esquema de desensibilización al día siguiente. Se reinició con un $\mathrm{VEF}_{1}$ del $73 \%$ del predicho para la paciente, con la misma dosis a la cual se presentó la reacción $(81 \mathrm{mg})$ y se continuó hasta que toleró una dosis de $650 \mathrm{mg}$ sin reacciones adversas. Se administró una dosis total acumulada de $1.343 \mathrm{mg}$, con buena tolerancia. Fue dada de alta y revisada a las 24 horas para evaluar reacciones tardías, las cuales no se presentaron. Continuó recibiendo una dosis de $650 \mathrm{mg}$ de ácido acetilsalicílico cada 12 horas y hasta la actualidad la ha tolerado.

\section{Discusión}

La desensibilización al ácido acetilsalicílico se considera una alternativa terapéutica con buena eficacia clínica y muy costo-efectiva en pacientes que, siendo intolerantes a AINE, cursan con dolor crónico o requieren el ácido acetilsalicílico para la profilaxis de una enfermedad cardiovascular y tromboembólica (11), en pacientes con asma y poliposis recurrente, los cuales no pueden ser controlados con polipectomías o cirugía de senos paranasales, y en mujeres con síndrome antifosfolípido durante el embarazo (9).

La desensibilización al ácido acetilsalicílico fue iniciada en 1922 por Widal y colaboradores (12), con la administración de dosis pequeñas de aspirina a pacientes intolerantes al ácido acetilsalicílico que padecían asma, hasta que la toleraban.

De un total de 107 pacientes con enfermedad respiratoria exacerbada por aspirina, se hizo seguimiento clínico a 65 sometidos a desensibilización al ácido acetilsalicílico y se compararon con un grupo control que no recibió ningún AINE. Se encontró que los pacientes que recibieron ácido acetilsalicílico tenían una significativa reducción en el número de hospitalizaciones, las consultas a urgencias, las infecciones de las vías respiratorias superiores y las cirugías por poliposis; igualmente, presentaron mejoría del olfato, en comparación con el grupo control; además, en ellos se redujó la necesidad de esteroides sistémicos (13).

En otro estudio a largo plazo de 65 pacientes con intolerancia al ácido acetilsalicílico, a quienes se les realizó desensibilización y recibían 1.300 mg diarios, se encontró una disminución significativa en el número de sinusitis infecciosas y en el uso de prednisona, con mejoría del olfato y de los síntomas de asma y rinitis. La necesidad de cirugía sinusal disminuyó de una cada tres años a una cada nueve años. Este estudio demostró el efecto benéfico a largo plazo de la desensibilización al ácido acetilsalicílico, en un período de seis años (14).

En un estudio posterior de 172 pacientes, realizado por Berges-Gimeno y colaboradores, se demostró un porcentaje de mejoría de $67 \%$ a los seis meses de tratamiento, el cual persistía por 1 a 5 años, con reducción en la aparición de sinusitis purulenta de cinco episodios por año, aproximadamente, a menos de la mitad (10).

Los esquemas tradicionales de desensibilización al ácido acetilsalićlico se han realizado durante tres días, administrando dosis progresivas hasta que se toleren $650 \mathrm{mg}$ sin presentar efectos adversos. El paciente debe seguir recibiendo dosis diarias de $650 \mathrm{mg}$ cada 12 horas $(3,15)$.

M. Castells (9) propone un protocolo de desensibilización rápida en 7 horas adaptado de otro propuesto por A. A. White (16) basado en la administración controlada de dosis progresivas de ácido acetilsalicílico a intervalos de 90 minutos hasta que el paciente tolere una dosis de 650 $\mathrm{mg}$ por vía oral, midiendo la función respiratoria mediante el $\mathrm{VEF}_{1}$ y considerando significativa una disminución mayor del $20 \%$ con respecto al valor basal $(9,17)$. En tal caso, se repite la dosis anterior hasta que no haya reacción y, luego, se continúa el esquema.

El protocolo anterior fue el usado por nuestro grupo en la desensibilización de las tres pacientes 
reportadas, aunque en el caso 1 sólo se requería una dosis total diaria de $100 \mathrm{mg}$ para el control del riesgo cardiovascular $(9,18)$. La terapia con ácido acetilsalicílico debe continuarse por tiempo indefinido para prevenir una posible reactivación de la intolerancia. Se considera que si hay una nueva exposición al medicamento después de suspender el ácido acetilsalicílico por más de 72 horas, puede presentarse nuevamente intolerancia al medicamento, con riesgo de una crisis de asma grave que ponga en peligro la vida del paciente (3).

Los riesgos inherentes a la desensibilización incluyen reacciones cutáneas, como urticaria, angioedema o ambas, crisis asmática, síntomas gastrointestinales y anafilaxia, las cuales se presentan en $20 \%$ de los pacientes, aproximadamente (3).

El mecanismo patogénico de las reacciones a AINE es complejo y no está totalmente entendido. La intolerancia a los AINE está vinculada a anormalidades en la vía del metabolismo delácido araquidónico, el cual se metaboliza por dos vías: la de la ciclooxigenasa y la de la lipooxigenasa. En condiciones fisiológicas, los metabolitos producidos por la vía de la ciclooxigenasa son la prostaglandina $\mathrm{E}_{2}\left(\mathrm{PGE}_{2}\right)$, la prostaciclina $\left(P G \mathrm{I}_{2}\right) \mathrm{y}$ los tromboxanos, mientras que, bajo condiciones de inflamación, los metabolitos también incluyen la prostaglandina $D_{2}\left(P G D_{2}\right)$ y la prostaglandina $F_{2}\left(P G F_{2}\right)$. Por la vía de la lipooxigenasa, el ácido araquidónico es metabolizado a leucotrieno $A_{4}$ $\left(\mathrm{LT}_{4}\right) \mathrm{y}$, además, es hidroxilado a leucotrieno $\mathrm{B}_{4}\left(\mathrm{LT} \mathrm{B}_{4}\right)$ o convertido en cisteinil-leucotrienos, como el leucotrieno $\mathrm{C}_{4}\left(\mathrm{LT} \mathrm{C}_{4}\right)$. La inhibición de la COX-1 desvía el metabolismo lejos de la producción de prostanoides protectores y hacia la vía de la lipooxigenasa y la producción de cisteinil leucotrienos. Otra hipótesis plantea que la estructura de la COX-2 es modificada por el ácido acetilsalicílico. Este cambio puede resultar en la generación de productos de la vía de la lipooxigenasa, con aumento en la actividad de los cisteinil-leucotrienos (figura 1).

La isoenzima COX-1 es la forma constitutiva de la enzima, presente en condiciones fisiológicas en casi todos los tejidos, aunque con aumento de su expresión en estómago, riñón, células endoteliales y plaquetas. La COX-1 protege la mucosa gastrointestinal contra la ulceración por medio de la producción de $P G E_{2}$, mientras que en las plaquetas la producción de tromboxano $A_{2}$ $\left(T \times A_{2}\right)$ y prostaciclina $\left(P G I_{2}\right)$ participan en la homeostasis y en la prevención de sangrados (8).

La isoenzima COX-2 es la forma inducible expresada en casi cualquier célula o tejido después de la estimulación por diferentes agentes, tales como citocinas, promotores tumorales y factores de crecimiento.

Los AINE clásicos (inhibidores de COX-1) bloquean ambas isoenzimas y son capaces de producir inflamación de la mucosa gástrica e intestinal, ulceración y sangrado. Por otro lado, los inhibidores de COX-2 son mejor tolerados por el sistema digestivo y tienen menos efectos adversos hematológicos que los AINE clásicos (19).

De acuerdo con su selectividad enzimática, los inhibidores COX-2 se han designado como preferenciales (nimesulide, meloxicam) y selectivos (celecoxib, rofecoxib, valdecoxib, parecoxib, etoricoxib, lumiracoxib).

A bajas concentraciones, el meloxicam y el nimesulide inhiben preferencialmente la COX-2; sin embargo, a altas concentraciones, bloquean también la COX-1. A bajas dosis, no inducen reacción cruzada en personas con enfermedad respiratoria exacerbada por ácido acetilsalicílico o urticaria crónica; sin embargo, con el incremento de la dosis, las reacciones cruzadas pueden ocurrir, evento que también se presenta con el acetaminofén. De otro lado, el meloxicam $y$ el nimesulide son excelentes haptenos y pueden unirse a proteínas transportadoras e inducir reacciones de hipersensibilidad mediadas por $\lg E(20)$.

El nimesulide fue introducido en Europa hace 16 años, aproximadamente, y hay estudios disponibles que tratan sobre su tolerabilidad en pacientes con antecedente de intolerancia a AINE, con un rango de reacciones cutáneas entre $3,3 \%$ y $25 \%(21,22)$. Los reportes de reacciones al meloxicam oscilan entre $1,3 \%$ y $20 \%(23,24)$. 


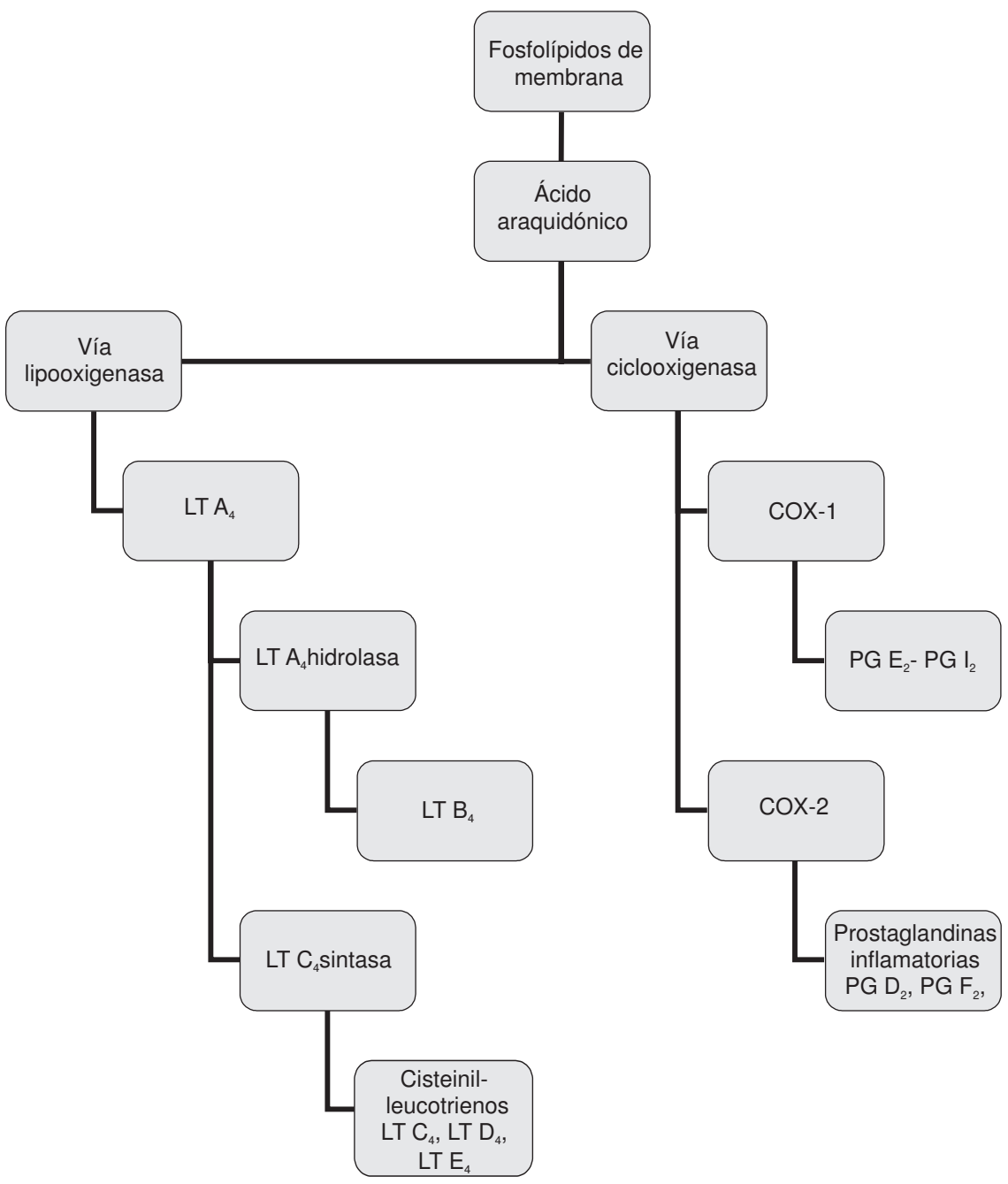

Figura 1. Metabolismo del ácido araquidónico.

Por muchos años, el acetaminofén ha sido el medicamento de elección para el tratamiento del dolor y la fiebre en pacientes con intolerancia a los AINE. Los estudios muestran un rango variable de reacciones entre $6,7 \%$ y $40 \%$. Esta discrepancia en los valores puede estar relacionada con las dosis utilizadas en las provocaciones y el hecho de que algunos pacientes toleran bajas dosis pero no un incremento de las mismas $(25,26)$.

Recientemente, se ha propuesto una nueva clasificación clínica usando la nomenclatura de la World Allergy Organization (2). Sin embargo, la clasificación clínica de la intolerancia a los
AINE más referenciada en la literatura es la vigente desde 2004, la cual divide la entidad en cuatro patrones clínicos (19).

Patrón respiratorio: incluye la enfermedad respiratoria exacerbada por el ácido acetilsalicílico, la tétrada de Samters (poliposis nasal, rinosinusitis, asma e intolerancia al ácido acetilsalicílico) y el asma inducida por el ácido acetilsalić́lico.

Patrón cutáneo: incluye urticaria y angioedema inducidos por AINE, urticaria y angioedema inducidos por múltiples fármacos, y urticaria y angioedema inducidos por un único medicamento. 
Patrón mixto: se presenta con síntomas cutáneos y respiratorios, que incluyen urticaria y angioedema asociados a tos, disnea, rinorrea, sibilancias, lagrimeo o irritación conjuntival.

Patrón sistémico: son reacciones anafilácticas de hipersensibilidad tipo I, generalmente observadas en casos de hipersensibilidad simple que toleran otros AINE no relacionados químicamente. Los anticuerpos lgE específicos para el alérgeno se unen a los mastocitos y a los basófilos, producen activación celular y liberan mediadores, con la consiguiente aparición de síntomas nasales, oculares, broncoespasmo, urticaria, dolor abdominal y colapso vasomotor. Teóricamente, cualquier AINE es capaz de funcionar como hapteno e inducir sensibilización.

La aproximación diagnóstica se basa en el cuadro clínico y su posible patogenia:

Reacciones alérgicas mediadas por Ig E: actualmente no existen reactivos estandarizados para pruebas cutáneas con AINE: su diagnóstico es esencialmente clínico. Se han desarrollado pruebas in vitro basadas en la liberación de sulfido-leucotrienos (cisteinil-leucotrienos) de leucocitos estimulados con factor $\mathrm{C} 5 \mathrm{a}$, pero no están disponibles fácilmente (8). Otra herramienta diagnóstica usada in vitro es la prueba de activación de basófilos o una prueba combinada para evaluar la activación de basófilos y determinar la liberación de sulfido-leucotrienos por basófilos después del reto con ácido acetilsalicílico o con otro AINE (3).

Reacciones tardías: las pruebas de parche son un método simple, fácil y seguro para el diagnóstico de reacciones tardías a AINE. Existen concentraciones estandarizadas de los AINE para su uso en los parches. La lectura del parche se realiza a las 48 y 72 horas $(27,28)$. La prueba de transformación de linfocitos mide la respuesta de proliferación in vitro de células T estimuladas por el medicamento. Esta prueba está disponible, por ahora, sólo en algunos laboratorios y centros especializados (29).

Reacciones no alérgicas: para reacciones cruzadas respiratorias y cutáneas, la prueba de referencia es la provocación oral realizada por personal experimentado en un centro especializado con acceso inmediato a equipos y medicamentos para manejo de reacciones (2). Las provocaciones con L-lisina ácido acetilsalicílico (L-ASA), nasales y bronquiales, también se usan para el diagnóstico $(30,31)$.

El manejo de los pacientes con intolerancia a los AINE depende de su historia clínica. Se deben clasificar según el tipo de sensibilidad, simple o cruzada, y el tipo de reacción, cutánea o sistémica.

Cuando el paciente presenta sensibilidad simple, se recomienda una prueba de provocación oral con un AINE de un grupo químico diferente al implicado. Si la prueba es negativa, puede recibir tratamiento con dicho AINE y evitar el medicamento sospechoso de producir la reacción. Si la prueba es positiva, se debe manejar como un caso de sensibilidad cruzada.

Cuando se clasifica como un caso de sensibilidad cruzada, se puede realizar una prueba de provocación oral con un inhibidor COX-1 débil o preferencial (acetaminofén, nimesulida, meloxicam, entre otros) o con un inhibidor COX-2 específico. Si la prueba es negativa, se procede a administrarlo al paciente y, si es positiva, se puede intentar una provocación oral con otro inhibidor COX-2 específico. Si esta última prueba es positiva, se deben evitar todos los AINE.

El paciente con antecedente de reacción anafiláctica por un único medicamento, amerita un trato especial. En este caso, se deben evitar todos los inhibidores COX-1 y realizar una prueba de provocación oral con un inhibidor COX-2 específico, en una unidad de cuidados intensivos; ésta se inicia con dosis bajas que se aumentan progresivamente hasta alcanzar la dosis terapéutica del medicamento, bajo estrecha vigilancia de los signos vitales y con disponibilidad de medicamentos y equipo de reanimación. Si el procedimiento es negativo, se puede usar el medicamento probado como alternativa analgésica y antiinflamatoria; si es positivo, se deben evitar todos los AINE o se puede realizar un procedimiento de desensibilización $(8,19)$. 


\section{Agradecimientos}

A la IPS Universitaria, una de las sedes de práctica clínica de la Facultad de Medicina de la Universidad de Antioquia, al personal de salud del Servicio de Alergología, al personal de la Unidad de Cuidados Intensivos tanto de la IPS Universitaria y de la Clínica León XIII, y a la terapeuta respiratoria Claudia Suárez.

\section{Conflicto de intereses}

Los autores no tienen ningún conflicto de intereses que declarar.

\section{Financiación}

Los procedimientos de desensibilización al ASA fueron autorizados y financiados por las respectivas Entidades Promotoras de Salud (EPS) de las pacientes.

\section{Referencias}

1. Demoly P, Hillaire-Buys D. Classification and epidemiology of hypersensitivity drug reactions. Immunol Allergy Clin North Am. 2004;24:345-56.

2. Sánchez-Borges M. Clinical management of nonsteroidal anti-inflammatory drug hypersensitivity. WAO Journal. 2008;1:29-33.

3. Jenneck C, Juergens $U$, Buecheler M, Novak N. Pathogenesis, diagnosis, and treatment of aspirin intolerance. Ann Allergy Asthma Immunol. 2007;99:13-21.

4. Cianferoni A, Novembre E, Mugnaini L, Lombardi E, Bernardini R, Pucci N, et al. Clinical features of acute anaphylaxis in patients admitted to a university hospital: an 11-year retrospective review (1985-1996). Ann Allergy Asthma Immunol. 2001;87:27-32.

5. Szczeklik A, Stevenson DD. Aspirin-induced asthma: advances in pathogenesis, diagnosis, and management. J Allergy Clin Immunol. 2003;111:913-21.

6. Faich GA. Adverse-drug-reaction monitoring. N Engl J Med. 1986;314:1589-92.

7. Settipane GA. Aspirin and allergic diseases: a review. Am J Med. 1983;74:102-9.

8. Sánchez-Borges M, Capriles-Hulett A, CaballeroFonseca F. NSAID-induced urticaria and angioedema: a reappraisal of its clinical management. Am J Clin Dermatol. 2002;3:599-607.

9. Castells M. Desensitization for drug allergy. Curr Opin Allergy Clin Immunol. 2006;6:476-81.

10. Berges-Gimeno MP, Simon RA, Stevenson DD. Longterm treatment with aspirin desensitization in asthmatic patients with aspirin-exacerbated respiratory disease. J Allergy Clin Immunol. 2003;111:180-6.

11. Gollapudi RR, Teirstein PS, Stevenson DD, Simon RA. Aspirin sensitivity: implications for patients with coronary artery disease. JAMA. 2004;292:3017-23.

12. Widal F, Abrami P, Lermoyez J. Anaphylaxie et idiosyncrasie. Presse Med. 1922;30:189-92.

13. Sweet JM, Stevenson DD, Simon RA, Mathison DA. Long-term effects of aspirin desensitization-treatment for aspirin-sensitive rhinosinusitis-asthma. J Allergy Clin Immunol. 1990;85:59-65.

14. Stevenson DD, Hankammer MA, Mathison DA, Christiansen SC, Simon RA. Aspirin desensitization treatment of aspirin-sensitive patients with rhinosinusitisasthma: long-term outcomes. J Allergy Clin Immunol. 1996;98:751-8.

15. Pfaar O, Klimek L. Aspirin desensitization in aspirin intolerance: update on current standards and recent improvements. Curr Opin Allergy Clin Immunol. 2006;6:161-6.

16. White AA, Stevenson DD, Simon RA. The blocking effect of essential controller medications during aspirin challenges in patients with aspirin-exacerbated respiratory disease. Ann Allergy Asthma Immunol. 2005;95:330-5.

17. Stevenson DD, Simon RA. Selection of patients for aspirin desensitization treatment. J Allergy Clin Immunol. 2006;118:801-4.

18. Silberman S, Neukirch-Stoop C, Steg PG. Rapid desensitization procedure for patients with aspirin hypersensitivity undergoing coronary stenting. Am J Cardiol. 2005;95:509-10.

19. Sánchez-Borges M, Capriles-Hulett A, CaballeroFonseca $\mathbf{F}$. The multiple faces of nonsteroidal antiinflammatory drug hypersensitivity. J Investig Allergol Clin Immunol. 2004;14:329-34.

20. Stevenson DD. Aspirin and NSAID sensitivity. Immunol Allergy Clin North Am. 2004;24:491-505.

21. Johansson SG, Hourihane JO, Bousquet J, Bruijnzeel-Koomen C, Reborg S, Haahtela $\mathrm{T}$, et al. A revised nomenclature for allergy. An EAACI position statement from the EAACI nomenclature task force. Allergy. 2001;56:813-24.

22. Andri L, Senna G, Betteli C, Givanni S, Scaricabarozzi I, Mezzelani P, et al. Tolerability of nimesulide in aspirinsensitive patients. Ann Allergy. 1994;72:29-32.

23. Kosnik M, Music E, Matjaz F, Suskovic S. Relative safety of meloxicam in NSAID-intolerant patients. Allergy. 1998;53:1231-3.

24. Nettis E, Di Paola R, Ferrannini A, Tursi A. Meloxicam in hypersensitivity to NSAIDs. Allergy. 2001;56:803-4.

25. Quiralte J, Blanco C, Castillo R, Delgado J, Carrillo T. Intolerance to nonsteroidal antiinflammatory drugs: 
results of controlled drug challenges in 98 patients. $J$ Allergy Clin Immunol. 1996;98:678-85.

26. Ruxrungtham K, Chantaphakul H, Tiyasatapon S, Phanupak P. Acetaminophen cross-sensitivity is common in Thai patients with aspirin/NSAIDs Sensitivity and may be life-threatening. J Allergy Clin Immunol. 2002;109:s141.

27. Zedlitz S, Linzbach L, Kaufmann R, Boehncke WH. Reproducible identification of the causative drug of a fixed drug eruption by oral provocation and lesional patch testing. Contact Derm. 2002;46:352-3.

28. Talhari C, Lauceviciute I, Enderlein E, Ruzicka T, Homey B. COX-2-selective inhibitor valdecoxib induces severe allergic skin reactions. J Allergy Clin Immunol. 2005;115:1089-90.

29. Pichler WJ, Tilch J. The lymphocyte transformation test in the diagnosis of drug hypersensitivity. Allergy. 2004;59:809-20.

30. Milewski M, Mastalerz L, Nizankowska E, Szczeklik A. Nasal provocation test with lysine-aspirin for diagnosis of aspirin-sensitive asthma. J Allergy Clin Immunol. 1998;101:581-6.

31. Nizankowska-Mogilnicka E, Bochenek G, Mastalerz L, Swierczynska M, Picado C, Scadding G, et al. EAACI/ GA2LEN guideline: aspirin provocation tests for diagnosis of aspirin hypersensitivity. Allergy. 2007;62:1111-8. 